\title{
Trophoblastic Disease During the Third Trimester of Pregnancy: Ultrasonic Diagnostic, Clinical Case and Perinatal Outcomes
}

\author{
Vitaly Tskhay*, Geyman A, Grebennikova E, Molgacheva E and Strelskaya 0 \\ Department of Perinatology, Krasnoyarsk State Medical University, Russia
}

*Corresponding author: Tskhay V, Department of Perinatology, Obstetrics and Gynecology, Krasnoyarsk State Medical University, Russia.

Received Date: December 07, 2018

Published Date: December 13, 2018

\section{Introduction}

Trophoblastic disease (TFD) considers rare tumors arising preferentially in reproductive age women. TFD is a relatively rare pathology and occurs at a rate of 1 in 22000 to 1 in 100000 pregnancies [1-3]. Trophoblastic tumors are always associated with pregnancy and can manifest both during pregnancy and after its completion (from several days to several years). The concept of "trophoblastic disease" unites several interconnected various forms of pathological condition of trophoblast: a hydatidiform mole, an invasive mole, choriocarcinoma, a placental bed tumor and an epithelioid trophoblastic tumor [2].

These tumours are rare, and they appear when cells in the womb start proliferating. Early diagnosis of GTD is essential for successful management while preserving fertility.

Malignant trophoblastic tumors (TT) are distinguished by high aggressiveness, rapid metastasis but, at the same time, high cure rates only with chemotherapy, even in the presence of distant metastases [4-6]. Various forms of TFD are considered by oncogynecologists as a single etiopathogenetic process [7]. The incidence of successful treatment of invasive forms of TFD, including choriocarcinoma, according to various authors, exceeds $80-90 \%$ [2]. After the cure of TFD, reproductive function is preserved in the absolute majority of young women.

The special properties of the ovarium, the effect of viruses (in particular, the influenza virus) on the trophoblast, immunological factors, increased hyaluronidase activity, chromosomal aberrations, and protein deficiency appear among the etiological prerequisites for TFD $[8,9]$.

Hydatidiform mole is the most common form of TFD, with an incidence rate of 1 case per 1000 pregnancies. Although, as a rule, the cystic is a benign condition, but in some patients, it can turn into an invasive form [10].

Hydatidiform mole, as one of the most common form, mainly develops in the first trimester of pregnancy and is characterized by the following clinical manifestations: vaginal bleeding (in 90\%); big size of the uterus that does not fit the period of gestation (in $50 \%$ cases); bilateral theca lutein cysts that exceed $8 \mathrm{~cm}$ in diameter (up to $40 \%$ of cases). The pregnancy with the hydatidiform mole can be complicated by toxicosis (uncontrollable vomiting), arterial hypertension, preeclampsia, signs of hyperthyroidism (hyperthermia, tachycardia, etc.), rupture of ovarian cysts, profuse bleeding $[2,7,11]$.

According to the authors of the majority published studies, the effect of a trophoblast tumor appears to be the most important and slightly studied in turns of association of the hydatidiform mole and normally developing singleton pregnancy [12-14]; the frequency of this form of TFD varies from 50 to $57 \%$. There are still no official recommendations and protocols for managing pregnancy in such situations, but some authors believe that pregnancy can be prolonged if no complications were observed, if the disease was diagnosed on time [15] and the probability of a live birth varies in the interval from 16 to $56 \%$ [16]. A fetus in such a condition usually has a normal karyotype, and there are approximately $25-40 \%$ chances of survival if the pregnancy is prolonged until the fetus reaches a time of viability [13].

The data presented by L. Wee in 2005 shows that the woman who decide to preserve her pregnancy with diagnosed TFD and having a live fetus should be aware by the doctor that she has only one chance out of four to give birth to a live child and approximately 
in $35 \%$ of the cases she may develop TFD syndrome after giving childbirth [17].

Here we are presenting a clinical case of similar pathology, associated with hydatidiform mole and a live fetus.

\section{Case Report}

A 30-year-old patient in the condition of the second pregnancy, though in the first upcoming birth. For 26 weeks of gestation she developed an increase in her blood pressure (up to $150 / 90 \mathrm{~mm}$ $\mathrm{Hg}$ ), proteinuria (up to $1 \mathrm{~g} / \mathrm{l}$ ) and had complains about her health condition. The pregnant woman was diagnosed with moderately severe preeclampsia and was administered to the appropriate drug treatment at the place of her residence. Since no effect was obtained, the patient was referred to our regional perinatal center (institution of the third level) at 31 weeks of gestation.

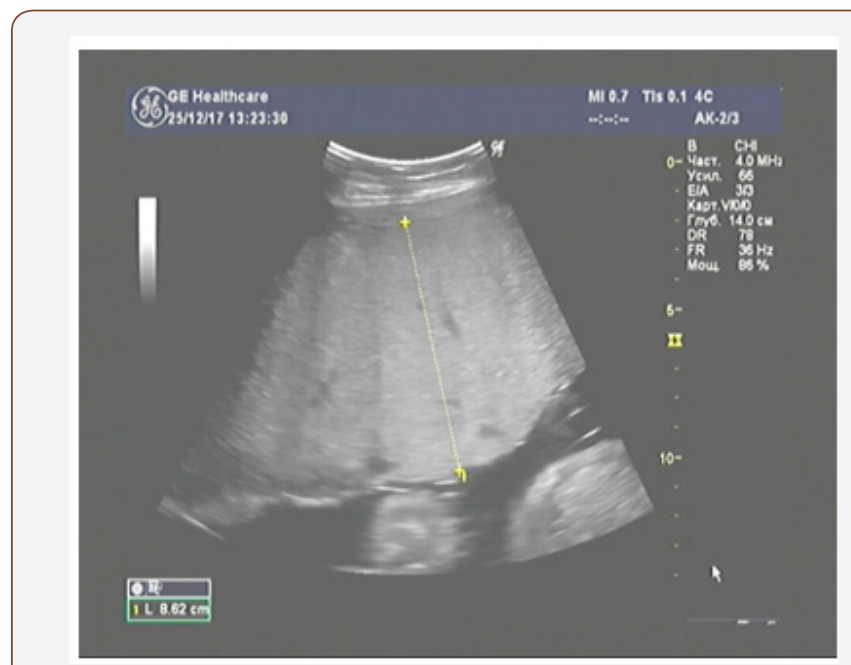

Figure 1: The echography of placenta (thick, edematous placenta).

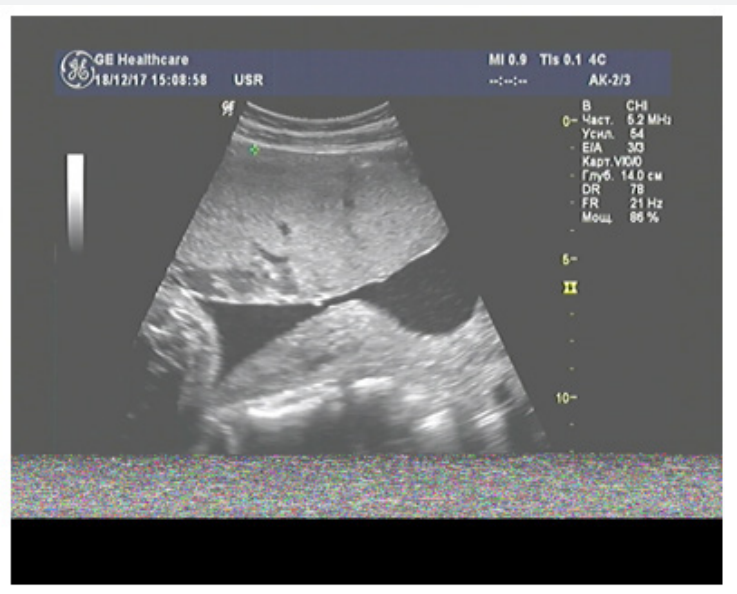

Figure 2: The echography of placenta (The presence of cystic changes in the placenta).

The results of ultrasound examination showed a single fetus in the uterus in the transverse position; in terms of its photometric parameters, the fetus fitted 31 weeks of gestation, the expectable estimated fetal weight was 1400 grams., a very thick placenta - up to $86 \mathrm{~mm}$ (Figure 1) with medium echogenicity, multiple small anechoic structures (Figure 2), moderately expressed oligohydramnios (amniotic fluid index - 72mm) and a short neck uterus according to the results of cervicometry ( $20 \mathrm{~mm}$ ). Another feature is large, multichamber, an echogenic, bulky ovarian formations on both sides of the uterus (Figures $3 \& 4$ ). The size of the right ovary was $88 \times 53 \times$ $83 \mathrm{~mm}$, of the left one $-91 \times 56 \times 82 \mathrm{~mm}$. Hemodynamic disorders of the uteroplacental circulation were not detected.

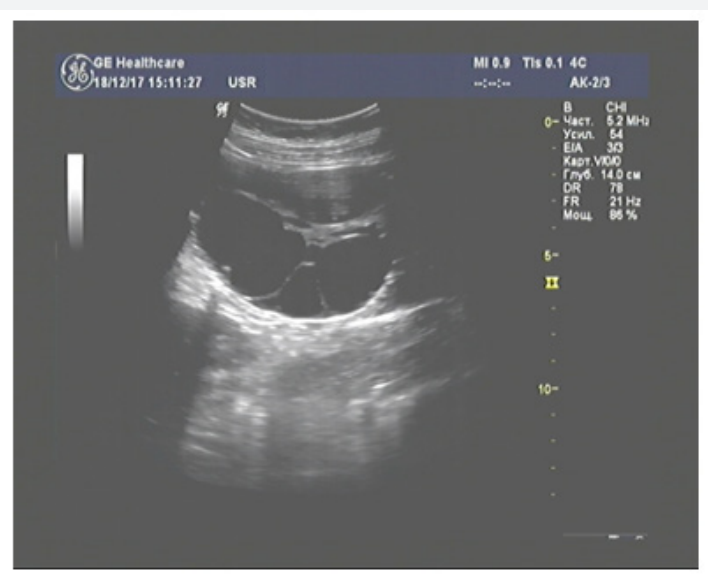

Figure 3\&4: Ovarial echography, presented by multi-chamber formations (Figure 3-Tumor-like formation of the right ovary; Figure 4- Tumor-like formation of the left ovary).

Considering the data of ultrasonic examination of the fetoplacental complex and the revealed pathological changes, we noticed the signs of a trophoblastic disease (thick, swollen, cysticaltered placenta and echo graphic changes in the ovaries that indicate the presence of theca lutein cysts).

To clarify the diagnosis, an additional assessment was performed - detection of HCG in serum, which showed the presence of high values of this indicator.

After receiving the results of HCG, which also confirmed the high probability of trophoblastic disease, a decision was taken about early delivery of the pregnant woman after a two-day course of glucocorticoids to prevent of respiratory disorders in the fetus by. Caesarean section was carried out on the $32^{\text {nd }}$ week of gestation.

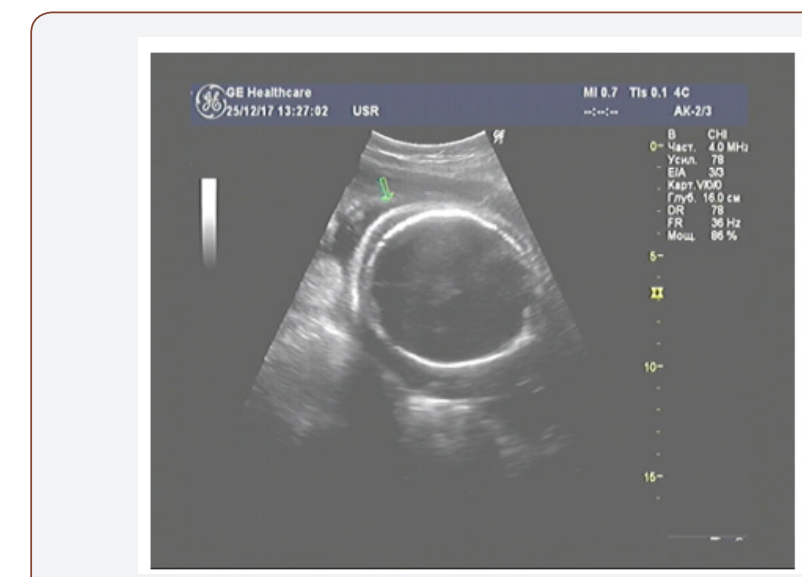

Figure 5: The image of a double contour of the fetus head.

On the day before the operation, a repeated ultrasonic examination showed the ultrasonic signs of fetal dropsy: a double 
contour of the head (Figure 5) and free fluid in the abdominal cavity of the fetus.

The outcome-live, premature female fetus born through a cesarean delivery, with pronounced signs of a non-immune dropsy of the fetus, weighing 1600 grams, $40 \mathrm{~cm}$ long. The Apgar score was 4 points on the 5 th minute after birth.

A large size of placenta, located on the front wall of the uterus, covered the most part of the uterine cavity, appeared to be the first sign of disorder. It was easily separated from the wall of the uterus in the course of operation. By visual examination there appeared a large and very thick placenta; swollen, with heterogeneous tissue in the form of a cluster of multiple cystic structures. The mass of the placenta was 1900 grams. The results of a subsequent histological examination of the placenta confirmed the presence of a trophoblastic disease - a partial hydatidiform mole. The ovaries matched the type of the calutein cysts (Figures 6\&7).

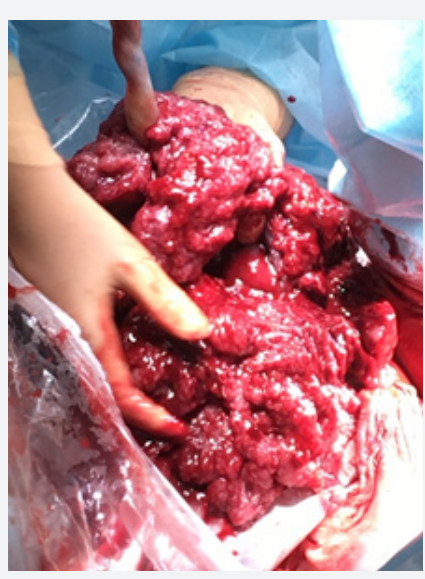

Figure 6: The image of placenta with particular signs of the pregnancy molar.

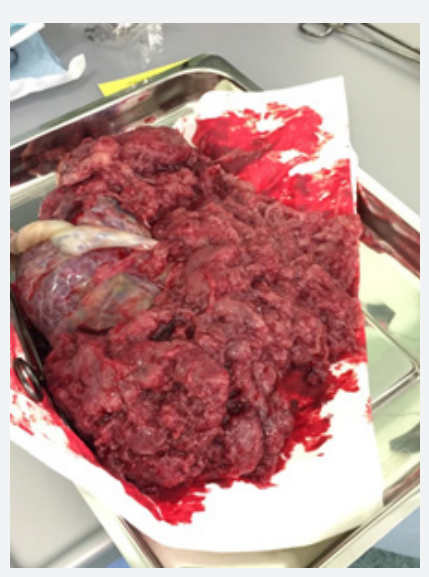

Figure 7: The image of placenta with particular signs of the pregnancy molar.

\section{Conclusion}

The progression of pregnancy in the third trimester with the simultaneous presence of a hydatidiform mole and a live fetus is an extremely rare medical condition (Figure 8). Therefore, today we do not have sufficient arguments to recommend early termination of the desired pregnancy, despite a certain risk for the mother. In the presented case, we made a decision to conduct early delivery on the basis of a sharp deterioration of the fetus (occurrence of dropsy) in a pregnant woman, who with a high degree of probability was supposed to have such a terrible complication like a trophoblastic disease.

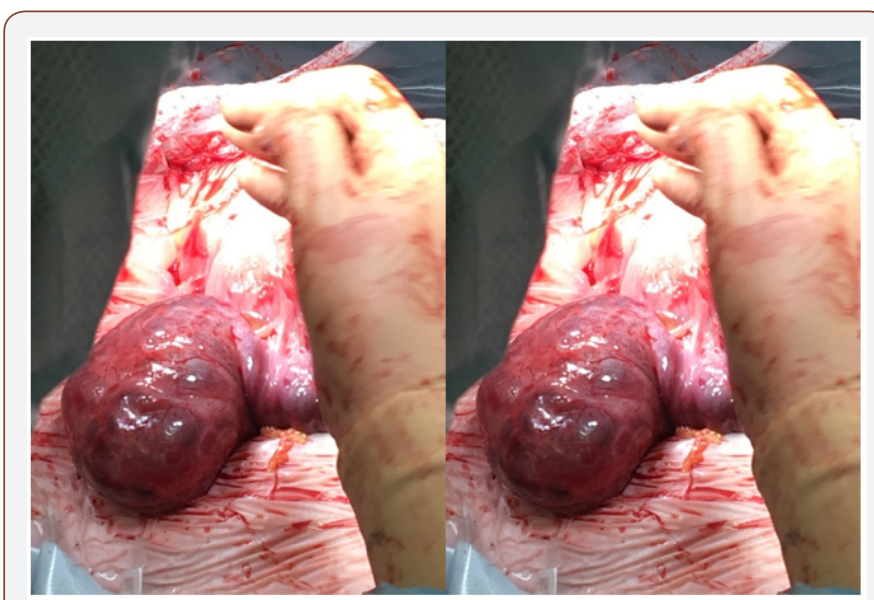

Figure 8: The image of the theca lutein cyst

\section{Acknowledgement}

None.

\section{Conflict of interest}

No Conflict of Interest.

\section{References}

1. Boubess I, Filali A, Benbrahim F, Ouassour S, Tazi M, et al. (2015) Twin pregnancy involving a molar pregnancy and living fetus with progression to invasive mole: about two cases. Pan Afr Med J 22: 24.

2. Biscaro A, Braga A, Berkowitz RS (2015) Diagnosis, classification and treatment of gestational trophoblastic neoplasia. Rev Bras Ginecol Obstet 37(1): 42-51.

3. Malhotra N, Deka D, Takkar D, Kochar S, Goel S, et al. (2001) Hydatiform mole with coexisting live fetus in dichorionic twin gestation. Eur J Obstet Gynecol reprod boil 94(2): 301-303.

4. Sita-Lumsden A, Short D, Lindsay I, Sebire NJ, Adjogatse D, et al. (2012) Treatment outcomes for 618 women with gestational trophoblastic tumours following a molar pregnancy at the Charing Cross Hospital, 2000-2009. Br J Cancer 107(11): 1810-1814.

5. Cavoretto P, Gentile C, Mangili G, Garavaglia E, Valsecchi L, Spagnolo D, et al. Transvaginal ultrasound predicts delayed response to chemotherapy and drug resistance in stage I low-risk trophoblastic neoplasia. Ultrasound Obstet Gynecol 40(1): 99-105.

6. Goldstein DP, Berkowitz RS, Horowitz NS (2015) Optimal management of low-risk gestational trophoblastic neoplasia. Expert Rev Anticancer Ther 15(11): 1293-1304.

7. Bohman YV (2012) Manual of Oncology. Book on Demand, p. 464

8. Lurain JR (2010) Gestational trophoblastic disease I: epidemiology, pathology, clinical presentation and diagnosis of gestational trophoblastic disease, and management of hydatidiform mole. Am J Obstet Gynecol 203(6): 531-539.

9. Shamshiri Milani H, Abdollahi M, Torbati S, Asbaghi T, Azargashb E (2017) Risk factors for hydatidiform mole: Is husband's job a major risk factor? Asian Pac J Cancer Prev 18(10): 2657-2662.

10. Lin LH, Bernardes LS, Hase EA, Fushida K, Francisco R (2015) Is Doppler ultrasound useful for evaluating gestational trophoblastic disease? Clinics (Sao Paulo) 70(12): 810-815.

11.Vencken PM, Ewing PC, Zweemer RP (2006) Epithelioid trophoblastic tumour: a case report and review of the literature. J Clin Pathol 59(12): 1307-1308. 
12. Anderson CK, Deiter RW, Motz MJ, Goldstein JA (1996) Complete hydatidiform mole with a coexistent healthy, viable fetus near term: a case report. J Reprod Med 41(1): 55-58.

13. Makary R, Mohammadi A, Rosa M, Shuja S (2010) Twin gestation with complete hydatidiform mole and a coexisting live fetus: case report and brief review of literature. Obstet Med 3(1): 30-32.

14. Polonsky A, Olteanu I, Ben-David M, Mamet J, Agranat A, et al. (2016) Viable triplet pregnancy coexisting with a complete molar pregnancy. Clin Case Rep 4(3): 247-249.
15. Bruchim I, Kidron D, Amiel A, Altaras M, Fejgin MD (2000) Complete Hydatiform mole and coexistent viable fetus: report of two cases and review of literature. Gynecologic oncology 77(1):197-202.

16. Massardier J, Golfier F, Journet D, Frappart L, Zalaquett M, et al. (2009) Twin pregnancy with complete hydatidiform mole and coexistent fetus: Obstetrical and oncological outcomes in a series of 14 cases. Eur J Obstet Gynecol Reprod Biol 143(2): 84-87.

17. Wee L, Jauniaux E (2005) Prenatal diagnosis and management of twin pregnancies complicated by a co-existing molar pregnancy. Prenat Diagn 25(9): 772-776. 